NASA Technical Memorandum 105649

AIAA-92-1960

\title{
Achieving Spectrum Conservation for the Minimum-Span and Minimum-Order Frequency Assignment Problems
}

(NASA-TM-105649) ACHIEVING SPECTRUM CONSERVATION FOR THE MINIMUM-SPAN AND MINIMUM-ORDER FREQUENCY ASSIGNMENT PROBLEMS (NASA) $28 \mathrm{P}$

\section{$1 N-17$ \\ 86817}

p. 28

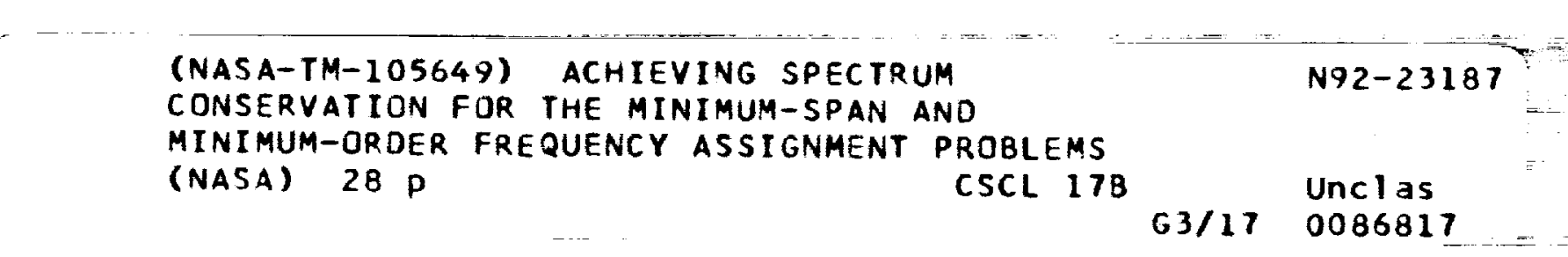

Ann O. Heyward

Lewis Research Center

Cleveland, Ohio

Prepared for the

14th International Communications Satellite Systems Conference sponsored by the American Institute of Aeronautics and Astronautics Washington, DC, March 22-26, 1992 



\title{
ACHIEVING SPECTRUM CONSERVATION FOR THE MINIMUM-SPAN AND MINIMUM-ORDER FREQUENCY ASSIGNMENT PROBLEMS
}

\author{
Ann O. Heyward \\ National Aeronautics and Space Administration \\ Lewis Research Center \\ Cleveland, Ohio 44135
}

\begin{abstract}
Effective and efficient solution of frequency assignment problems assumes increasing importance as the radiofrequency spectrum experiences ever-increasing utilization by diverse communications services, requiring that the most efficient use of this resource be achieved. The research presented explores a general approach to the frequency assignment problem, in which such problems are categorized by the appropriate spectrumconserving objective function, and are each treated as an $\mathrm{N}$-job, $\mathrm{M}$-machine scheduling problem appropriate for the objective. Results obtained and presented illustrate that such an approach presents an effective means of achieving spectrum-conserving frequency assignments for communications systems in a variety of environments.
\end{abstract}

\subsection{Introduction}

The general frequency assignment problem may be stated as follows: given a number of nodes in a set of communications networks, and constraints imposed by the need to eliminate unacceptable levels of a variety of types of interference between communications links, determine the assignment of frequencies (channels) to each node that achieves acceptable performance relative to one or more selected criteria. This general problem statement encompasses a range of problems of interest to the communications planner. One such problem is the need to assign sets of frequencies or channels to the transponders of a collection of communications satellites serving a variety of geographical areas on the Earth, so that allowable levels of co-channel and adjacent-channel interference are not exceeded, all requirements for channels are met, and the minimum number of channels is utilized. Alternatively, in frequency division multiple access (FDMA) satellite networks, dynamic channel assignment to network nodes accessing a single satellite may be required, while preserving as much continuous spectrum as possible to respond to fluctuating demands.
There are a number of insights to be gained from previous research that may be applied to the development of an approach to the frequency assignment problem. The frequency assignment problem is NP-hard; it is unlikely that a computational procedure can be devised which will find a truly optimum assignment within reasonable computation time for large, complex problems ${ }^{15,16}$. Certain classes of frequency assignment problems are equivalent to graph-coloring problems ${ }^{18,39}$. Graph-coloring approaches therefore provide some strategies which may be extrapolated to specific frequency assignment problems, particularly with regard to ordering of frequency assignment requirements to be satisfied $^{5,6,7,8,9,21,26,27,31,34,36,37,39}$. A number of heuristic approaches to the frequency assignment problem have also been developed. Such approaches often exploit specific assignment strategies in order to minimize spectrum use or, alternatively maximize spectrum conservation. $1,2,4,10,11,12,13,20,23,24,25,28,29,30,32,33,38$.

An approach particularly relevant to the research presented in this paper is that of Ignizio ${ }^{19}$, which uses a generalized goal-programming approach to the minimal interference, multicriterion, $\mathrm{N}$-job, 1-machine scheduling problem. Examination of this approach implies that a more general, $\mathbf{N}$-job, M-machine scheduling approach displays significant promise as a frequency assignment technique.

High demands are placed on virtually all portions of the frequency spectrum that are currently in use for communications applications. Thus, frequency/channel assignment procedures should seek spectrum conservation. As previous researchers have noted, alternative definitions of spectrum conserving frequency assignment are possible ${ }^{18}$. A minimum-order frequency assignment (utilizing the minimum number of discrete frequencies) may be sought; this objective is desired in circumstances dictating conservation of the maximum number of discrete frequency slots for future 
assignment. Alternatively, a minimum-span assignment (with minimum difference between highest-valued and lowest-valued assigned frequencies) is desired when the maximum length continuous portion of the spectrum should be conserved for future assignment. These objectives are conflicting: use of a greater frequency span may be required to achieve a minimum-order assignment, and, conversely, use of a higher number of frequencies may be required to achieve a minimum-span assignment. These conflicting objectives require different modelling and solution approaches, but both objectives are accommodated within the theoretical framework of $\mathrm{N}$-job, $\mathrm{M}$-machine scheduling problems.

Two N-job, M-machine scheduling models of the frequency/channel assignment problem were developed, corresponding to minimum-span and minimum-order frequency assignment objectives, and were applied to problems of varied size and complexity. Results indicate circumstances under which use of each model is suitable. For the model corresponding to minimum-span frequency assignment, results also indicate circumstances under which each of two requirements-ordering techniques are most effective. Finally, both models and associated requirements-ordering techniques are demonstrated to result in spectrum-conserving frequency assignments, thus presenting an effective general approach for solution of minimum-order and minimumspan frequency assignment problems via $\mathrm{N}$-job, $\mathrm{M}$ machine scheduling.

\subsection{Scheduling Models of the Frequency Assignment Problem}

Two N-job, M-machine scheduling models of the frequency/channel assignment problem are presented below, corresponding to the conflicting objectives of minimum-span and minimum-order frequency assignment, respectively. The first model is a scheduling analog of the minimum span frequency assignment problem - the Minimum Makespan Model. The second model is a scheduling analog of the minimum order frequency assignment problem -the Minimum Resource Model. The first model is applicable to frequency assignment in portions of the spectrum allocated to more than one service, whose frequency assignments cannot be interleaved or cannot overlap, since the maximum-length continuous portion of the spectrum is conserved for future assignment. The second model is applicable to frequency assignment in portions of the spectrum allocated to only one service, where conservation of the maximum number of discrete frequency slots for future assignment is desired.

\subsection{The Minimum Makespan Model}

Minimization of the makespan, or total time, required to complete a given series of jobs or operations within a schedule is directly analogous to minimizing frequency span, if available frequency bandwidth is mapped into a finite time interval. The minimum time needed to complete all jobs in the schedule will be equivalent to use of the minimum frequency span to complete the assignment.

A schedule will determine specific start and stop times for particular jobs, which consist of a series of distinct operations to be performed on different machines. Machines represent distinct nodes in a communications system where operating frequencies are required; each node may require a different number of operating frequencies. An operation within a job represents assignment of one operating frequency to one node; each job, therefore, will consist of the assignment of an operating frequency to each of a series of nodes. Interference protection requirements between operating frequencies at different nodes are represented as time separations between operations within the same job, or different jobs, under the assumption that all interference protection requirements between nodes may be expressed as frequency separation requirements.

To recover a frequency assignment from solution of a scheduling problem, the resulting schedule must translate directly to a frequency assignment. To construct a one-to-one mapping of frequency to time, let time interval $(0, \ldots, T)$ represent the interval $(0, \mathrm{BW})$ where $\mathrm{BW}$, the total frequency bandwidth available, is the difference between the lowest frequency available and the highest frequency available, e.g. $F_{\max }-F_{\text {min }}$. The interval $(0, \mathrm{BW})$ is discretized by selecting a small portion of the available bandwidth, $\mathrm{F}_{\text {norm }}$, so that the total interval $(0, \mathrm{BW})$ contains a reasonable number of discrete units that each approximate a unit of frequency separation required between assignments. In order to construct channel assignments, $F_{\text {norm }}$ must be an integer divisor of the channel bandwidth. The interval $(0, B W)$ may then be discretized as $\left(0,1^{*} F_{\text {norm }}, 2^{*} F_{\text {norm }}, \ldots, B W\right)$; discrete times in the interval $(0, T)$ are then:

$$
x_{i}=\frac{f_{i}-F_{\min }}{F_{\text {norm }}}+1
$$

where $f_{i}$ is in $\left(F_{\min }, F_{\max }\right)$, the range of frequencies 
available for assignment. Assigned frequency values may be recovered from the scheduling problem solution by inverting (1):

$$
f_{i}=\left(x_{i}-1\right) F_{\text {morm }}+F_{\min }
$$

where $x_{i}=(0, \ldots, T)$.

The transformation from the frequency interval $\left(F_{\min }, F_{\max }\right)$ to the discretized time interval $(0, T)$ is illustrated in Figure 1.

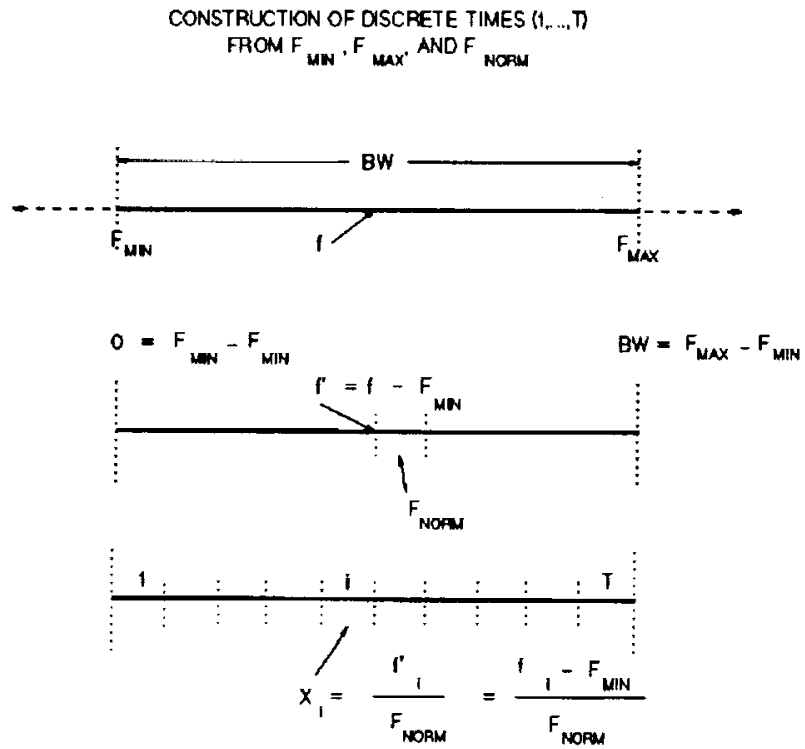

Figure 1

The frequency assignment resulting from the scheduling problem solution must determine operating frequencies for a collection of nodes in a network so that the required number of frequencies for each node are found and frequency separation constraints between distinct nodes are satisfied. Each node may require a different number of operating frequencies, since each node may have to accommodate a different quantity of traffic. Primary constraints are those needed to protect signals from co-channel and/or adjacent channel interference, though constraints designed to limit intermodulation interference would not be difficult to include in the model.

Consider a collection of nodes, each requiring assignment of a specified number of operating frequencies. Each node may be represented as a machine, upon which one or more processing tasks or operations are to be completed. If each node, $S_{i}, i=(1$,
$2, \ldots, M)$ is represented as a separate machine, $M_{j}, i=$ $(1, \ldots, M)$, the task of assigning an operating frequency to any subset of the set of nodes is equivalent to a job, consisting of $\mathrm{M}$ or fewer operations, each of which must be performed on a different specified machine. The series of jobs to be performed is determined by examining nodes in sequence. Assuming that all nodes require at least one operating frequency, the first job will have $\mathbf{M}$ operations, to be performed on machines $1, \ldots, M$. If some nodes require more than one operating frequency, a second job is constructed which will have an operation associated with each node (machine) requiring an additional operating frequency. In this manner, a series of jobs $\left(J_{1}, J_{2}, \ldots, J_{n}\right)$ is constructed, where $\mathbf{n}$ is the maximum number of operating frequencies required by any node. Each "job" will be represented by a column vector of Os and $1 \mathrm{~s}$; a "1" in the $\mathrm{i}$-th row indicates that the job requires an operation to be performed on the i-th machine (i.e., that a frequency is to be assigned to the $\mathrm{i}$-th node). The sequence of job vectors is lexicographically ordered, as a direct result of the construction sequence, effectively creating a series of subsets of the communications network nodes. The first subset will consist of all nodes requiring at least one operating frequency - or, the complete set. The second subset will consist of all nodes requiring at least two frequencies; the third subset, all those requiring three or more. The final subset consists of the node(s) requiring the maximum number of frequencies; therefore, the total number of jobs will equal the maximum number of frequencies required by any node. Each subset will contain at most as many elements as its predecessor. Thus, each job vector will have a non-increasing number of operations.

Operations within each job are ordered by the machine on which they must be performed. As a result, the scheduling model is simplified since additional variables are not required to associate individual operations with particular machines. The scheduling model is further simplified in that jobs are scheduled in the same order as constructed; permutations of job ordering need not be considered.

Constraints affecting feasible operating frequencies at different sites are implemented via frequency separation requirements. The scheduling analog of a frequency separation requirement - to avoid co-channel or adjacent channel interference between operating frequencies assigned to different nodes - is a time separation, expressed as number of discrete time intervals required between operations to be performed 
on different machines within the same job. A frequency separation requirement between operating frequencies that may be assigned for use by nodes $j$ and $k$ will translate to a time separation requirement $t_{j k}$, determining the feasible start time for the k-th operation of any job, relative to the scheduled start time for operation $\mathrm{j}$. If the start time for the $\mathrm{j}$-th operation of a given job is $x_{i j}$, operation $k$ of that job and any subsequent job cannot start before time $\mathrm{x}_{\mathrm{ij}}+\mathrm{t}_{\mathrm{jk}}$. This concept is illustrated in Figure 2.

OPERATION SCHEDULING SUBJECT TO SEPARATION CONSTRAINTS

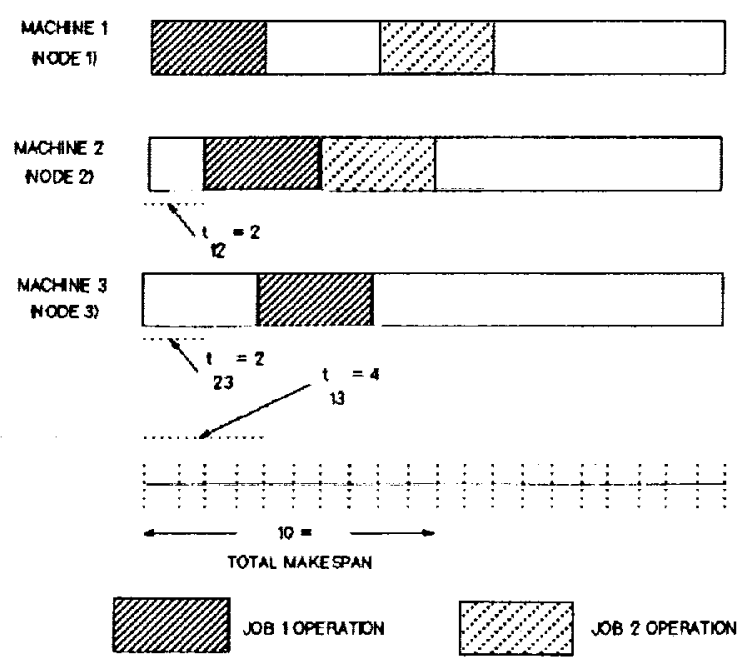

Figure 2

The question of machine (node) ordering has not yet been dealt with. The total makespan achieved for the schedule will not be affected by the ordering of jobs. Since the earliest available start time on each machine is determined by interference constraints, it is the ordering of nodes or machines that impacts the total makespan by determining the start times $\mathrm{x}_{\mathrm{ij}}$ feasible for all operations on all machines. If operations on the machine requiring the maximum number of operations can be started at an earlier time, total makespan to complete all operations on that machine will be reduced. The investigation of alternative orderings for machines (nodes) resembles the node-ordering aspect of graphtheoretic implementations of the frequency assignment problem. Optimal ordering of machines (or nodes) will result in minimized makespan, as illustrated in Figure 3, in which schedules resulting from machine (node) orderings applied to the example of Figure 2 are presented.

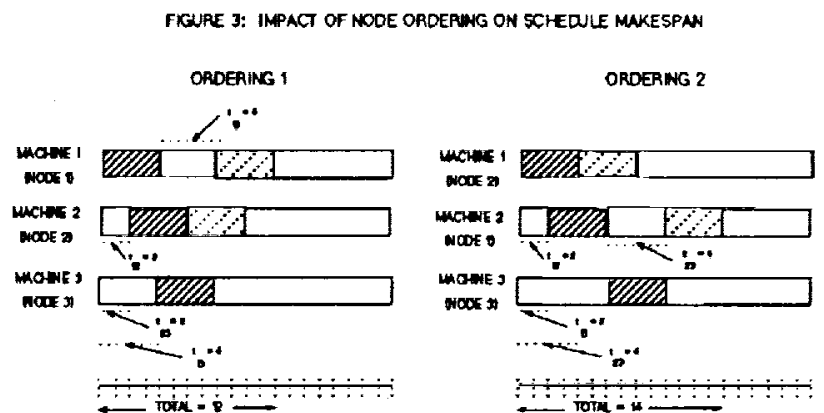

Figure 3

Each ordering of machines or communications network nodes will have an associated scheduling problem which will determine a frequency assignment. Given an identification of nodes with machines (a node ordering),

Minimize

$$
\left[\max _{k}\left(x_{n k}+r_{n k}\right)\right]
$$

Subject to:

$$
\begin{gathered}
x_{p k}-x_{i k} \geq r_{i k k} p>i, \\
x_{i k}+t_{i k k} \leq x_{i k} \\
x_{i k} \leq x_{i k}-t_{i k h}, h>k, \\
x_{i k}+r_{i k} \leq T \\
x_{i k} \geq 1
\end{gathered}
$$

where:

$$
i=(1, \ldots, n), \quad k=(1, \ldots, M)
$$

M = total number of machines (nodes);

n = total number of jobs (sequence of frequency assignments that must be made);

$\mathbf{T}=$ latest discrete time interval at which any operation may conclude on any machine;

$\mathrm{x}_{\mathrm{ik}}=$ start time interval of job $\mathrm{i}$ (i.e., an operation associated with job $i$ ) on machine $k$ (decision variables); 
$t_{i k h}=$ offset, in discrete time units, from the start of operation $k$ of job $i$ on machine $k$ that must elapse before a subsequent operation may be started on machine h;

$\mathbf{r}_{\mathbf{i k}}=$ required processing time in discrete intervals, operation $k$ of job $i$ (which is performed on machine $\mathbf{k}$ ).

Since $i=(1, \ldots, n)$ and $k=(1, \ldots, M)$, there will be a total of $M n$ decision variables $x_{i k}$, the values of which will determine the schedule. Required frequency separations are enforced by constraints (5) and (6). While this set of constraints appears disjunctive, the disjunction is resolved by both nonnegativity and required processing time constraints. Any operation associated with any job will have an associated processing time, which must be taken into account when scheduling operations associated with different jobs on the same machine. Constraint (4) ensures that the conclusion of operation $i$ precedes the start of operation $p$ for operations performed for different jobs on the same machine. These constraints are considerably reduced in number and complexity by processing jobs in the order in which they are created. If this were not the case, disjunctive precedence constraints would be required. If the job precedence relation were not already established, an "either-or". disjunctive ordering relationship would have to be accommodated in precedence constraints developed - i.e., precedence constraints would have to be included for the case $\mathrm{p}<\mathrm{i}$, as well; additionally, the problem would be made more complex by virtue of the need to activate the correct constraints depending on the job ordering selected.

Since the frequency assignment derived for the collection of nodes must lie within the available frequency bandwidth, constraint (7) requires that all operations, for all jobs, on all machines, conclude within the total available time, T. Constraint (8) ensures that no operations start before the beginning of the total time interval available.

The objective is minimization of schedule makespan i.e., minimization of the difference between the earliest start time scheduled for any operation of any job, and the latest completion time for any operation of any job. Jobs are ordered lexicographically; therefore, the last operation of the last job scheduled will have the latest start time. If variable processing times are permitted, it cannot be assumed that the operation with the latest start time is also the operation with the latest conclusion time. Therefore, the objective is expressed as (8), minimizing over all machines $k=(1, \ldots, M)$, the latest completion time of any operation of the last job scheduled.

\section{2,2 The Minimum Resource Model}

The Minimum Resource Model represents an alternative view of spectrum conservation, seeking minimization of the number of distinct frequencies utilized to successfully accomplish an assignment. The scheduling case considered here concerns use of machine resources, where each machine represents a frequency, minimizing the number of machines used to process operations to be scheduled is equivalent to using the minimum number of frequencies to accomplish a successful assignment.

The total number of machines available for processing jobs will be determined in part by the total frequency bandwidth within which frequencies must be assigned, and how the bandwidth is divided. If channels of a specified bandwidth are required, an upper bound on the total number of active machines required can be initially determined by the number of channels accommodated by the total available bandwidth. The same is true for discrete frequencies, as opposed to channels. This formulation assumes that either channels or discrete (as opposed to continuous) frequencies are assigned.

Since there are a specific number of nodes in the communications network, and each node requires a specific number of operating frequencies, this bound can be reduced. Assuming that each of the frequencies assigned at a node must be different, an alternative upper bound for the required number of active frequencies, or machines, can be determined. If sufficient bandwidth exists to satisfy these requirements, the entire bandwidth may not be needed in constructing a set of active machines for the scheduling problem. At most, the number of active machines required to provide a separate distinct frequency for each requirement of each of the nodes is needed. Therefore, an initial bound on the total number of active machines (frequencies) required is determined by summing over all nodes the number of operating frequencies required by each, e.g.

$$
M_{A}=\sum_{i=1}^{n} n_{i}
$$

where $n_{i}$ is the number of operating frequencies 
required by node $i, i=(1, \ldots, n)$, where $n$ is the total number of nodes. This initial upper bound on the number of active machines required may not be sufficient as an initial upper bound on the total number of machines (frequencies), both active and inactive, that must be made available to complete a schedule satisfying all constraints. It may be possible to reduce the number of active machines required, which may leave selected machines idle. The need to enforce frequency separations may also require that additional idle machines be made available. An initial upper bound can be determined for the total number of machines that must be made available, by counting the number of constraints, $s_{i}$, associated with each node $i$. Since there may be multiple frequencies required per node, $s_{i}$ must be multiplied by the number of requirements for the node, $\mathrm{n}_{\mathrm{i}}$. Summing over all nodes results in the upper bound

$$
M=\sum_{i=1}^{n} s_{i} n_{i}
$$

Jobs, operations, time intervals, and constraints are characterized in a manner consistent with the objective of minimizing resource use, i.e., use of separate machines to perform operations, where each machine corresponds to a discrete frequency or channel. An operation on a machine represents assignment of a frequency to a given node. The requirement of a given number of separate operating frequencies by a given node cannot be satisfied if two operations identified with the same node are performed on the same machine; this would mean attempting to satisfy two frequency requirements at the same node with the same frequency. Each operation associated with the same node must be performed on a different machine. Accordingly, each job, consisting of a set of tasks to be performed on a different machine, represents a complete frequency assignment for a single node. The number of jobs to be scheduled equals the number of nodes in the communications network, i.e, $n$. The number of operations required by each job will correspond to the number of frequencies required by the node represented by the job; i.e., if jobs $J_{1}, \ldots ., J_{n}$ are associated with nodes $i=(1, \ldots, n)$, job $J_{i}$ will require $n_{i}$ operations, where $n_{i}$ is the number of frequencies required by node $i$. Since more machines may be available for use than are actually necessary to perform all jobs, it cannot be assumed that each operation within $a$ job is automatically identified with the machine on which it must be performed. The minimum resource scheduling model requires decision variables for this purpose.
An upper limit on total time available, within which all jobs must be completed, is also required. In the Minimum Makespan Model, time corresponded to frequency and an upper limit on available frequency bandwidth translated to a limit on available time. This is not true here, as machines represent frequencies; limitations on frequency resources imply limitations on the number of available machines. However, discrete time intervals and an upper limit on available time can be defined in a way that benefits the structure of the Minimum Resource Model.

Any operation, for any job, on any machine, will be assumed to require unit processing time. Since a job consists of a complete frequency assignment to one of $N$ nodes, and no node may use the same frequency twice, no job may use the same machine twice. Each operation requires unit processing time, and operations associated with at most $n$ different jobs can be performed on each machine, so an upper limit of $n$ time units available exists. It will be required that all operations associated with a single job be performed simultaneously on different machines; operations associated with different jobs cannot be performed simultaneously. Therefore, on any machine, the time interval associated with an operation will provide an index of the job that is scheduled. Decision variables associating jobs and start times are not required. Structuring time intervals as described simplifies counting the number of usages of each "active" machine, which simplifies calculating the objective function.

Once again, constraints of primary concern are frequency separation constraints limiting co-channel and adjacent-channel interference. Restrictions on frequencies allowable for different nodes will imply that performance of a specific operation on a given machine will prohibit the use of a specific set of machines for operations associated with other jobs. Let $x_{i j}$ represent the index of the machine to which operation $j$ of job $i$ is assigned. Since job $\mathrm{i}$ represents a complete frequency assignment for node $i$, let frequency separation requirements between frequencies utilized for node $i$ and all other nodes $\mathbf{k}$ be represented as multiples of a discrete frequency interval. Each machine corresponds to a discrete frequency range. Thus, frequency separation requirements between frequencies for nodes $i$ and $k$ are expressed as differences in machine index separating feasible machines for jobs $i$ and $k, r_{i k}$. Use of machine $x_{i k}$ for the $j-t h$ operation of job $i$ implies that the machine with index $x_{i j}+r_{i k}$ is the next feasible machine on which a job $k$ operation may performed. 
The objective is to minimize the number of active machines required to accomplish the assignment, while meeting all such constraints.

Figure 4 illustrates the concept that different schedules, for the same set of nodes and constraints, may require different numbers of active machines out of a total number of machines available.

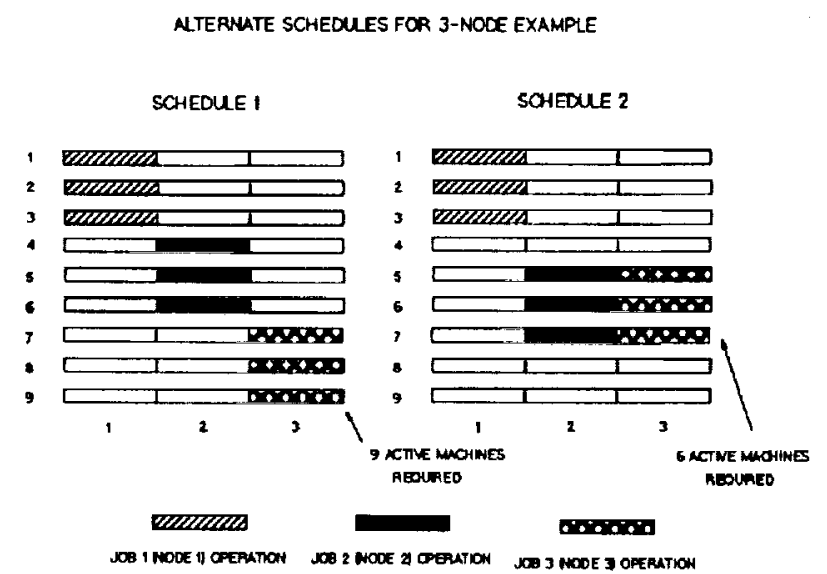

Figure 4

Once an upper bound on the total number of both active and inactive machines has been determined, the following minimum resource scheduling problem can be solved:

Minimize:

$$
\sum_{m=1}^{M} \sum_{i=1}^{N} y_{i m}-\sum_{m=1}^{N} \max \left[0,\left(\left(\sum_{i=1}^{N} y_{i m}\right)-1\right)\right]
$$

Subject to:

$$
\begin{gathered}
x_{i j} \leq M \\
x_{b p} \geq x_{i j}+r_{i k} \\
\sum_{m=1}^{N} y_{i m}=n_{i}
\end{gathered}
$$

$$
\begin{gathered}
\sum_{i=1}^{n} y_{i m} \leq n \\
y_{i m} x_{i j}=m \\
x_{i j} \geq 0 \\
y_{i m} \geq 0
\end{gathered}
$$

where

$$
\begin{aligned}
& i=(1, \ldots, n), j=\left(1, \ldots, n_{i}\right), m=(1, \ldots, M), p=\left(1, \ldots, n_{k}\right) \\
& M=\text { total number of machines available; } \\
& \text { n = total number of jobs (nodes); also, total }
\end{aligned}
$$

The variables $\boldsymbol{r}_{\mathrm{ik}}$ implement frequency separation constraints. The $\mathrm{x}_{\mathrm{ij}}$ provide a method to assign time slots to operations on each machine. The variables $y_{i m}$ provide a way to determine the number of operations utilizing each machine.

Constraint (13) determines feasible machine indices for all operations. Constraint (14) requires that all jobs be performed on some machine. Constraint (15) requires that no more than $n$ operations, for $n$ jobs, be performed on the same machine. These constraints also ensure that no two operations associated with the same job will be performed on the same machine; in other words, the same frequency will not be used to provide two frequency assignments at the same node. In the 
definition of decision variables $y_{i m}, y_{i m}$ equals 1 if $x_{i j}$ equals the machine index $\mathrm{m}$. Constraint (16) implements this requirement. Constraints (17) and (18) require that decision variables $x_{i j}$ and $y_{i m}$ be nonnegative.

The objective is minimization of the total number of active machines utilized to perform all jobs. The total number of active machines is equivalent to the total number of uses of all machines by all operations of all jobs, minus the uses over and above the first use of each machine by an operation of any job. The number of total uses of all machines is

$$
\sum_{m=1}^{M} \sum_{i=1}^{n} y_{i m}
$$

The uses of any individual machine may be expressed as

$$
\sum_{i=1}^{n} y_{i m}
$$

The number of uses of each machine over and above the first use, is:

$$
\left(\sum_{i=1}^{n} y_{i m}\right)-1
$$

The number of uses of any machine (active or idle) is given by

$$
\max \left[0,\left(\left(\sum_{i=1}^{n} y_{i m}\right)-1\right)\right]
$$

The objective is thus to minimize

$$
\sum_{m=1}^{M} \sum_{i=1}^{n} y_{i m}-\sum_{m=1}^{M} \max \left[0,\left(\left(\sum_{i=1}^{n} y_{i m}\right)-1\right)\right]
$$

A secondary objective is systematic reduction of the total number of active and inactive machines required for the assignment; in other words, utilizing the minimum number of frequencies within a minimal makespan. Accordingly, the bound $M$ on the total number of machines can be iteratively reduced, and a series of problems for different bounds on $x_{i j}$ can be solved.

\section{Solution Techniques}

In the Minimum Makespan Model, the decision variables, $x_{i j}$, represented possible start times for $i=$ $(1, \ldots, n)$ jobs on $\mathrm{M}$ machines. The scheduling problem is solved by enumerating at most two start times for any operation of any job. In order to schedule any operation $h$ of any job $i$, at most two possible start times must be considered, since constraints (4), (5), (6), (8) must be satisfied:

$$
x_{i-1, k}+r_{i-1, h} \quad(1 \text { if } i=1)
$$

and, if (25) is infeasible

$$
\max _{k+h}\left(x_{i k}+t_{i k k}\right)
$$

By explicitly considering at most two start times for each operation of each job, we implicitly enumerate all feasible schedules and minimize makespan by scheduling at the earliest feasible start time for each operation of each job. Evaluation of these two start times suffices to assess the entire range of possible values $(1, \ldots, \mathrm{T})$ for all $\mathbf{x}_{\text {in }}$.

As discussed in section 2.0, ordering of nodes requiring assignment of communications frequencies impacts the total schedule makespan. For $n$ sites, n! possible orderings are possible; exhaustive examination of all orderings is impractical. Therefore the ordering strategy to reference nodes to machines should be developed from insight into the frequency assignment problem. It is also desirable to examine more than one ordering strategy for a given problem to determine which strategy yields minimum makespan; it is possible that one strategy may produce better results for a given problem than an alternate strategy. Two ordering strategies were developed for the solution of the Minimum Makespan Model.

Completing the "most difficult" assignment first, followed by assignments of decreasing difficulty, has intuitive appeal in that it is logical to assume that the assignments that are the least difficult are the most flexible in their requirements, and will have fewer feasible assignments prohibited by virtue of preceding assignments made. Frequency separation requirements between nodes may be listed in a matrix, in which each pair of nodes is represented by a row and column index. Each matrix (row, column) entry represents the frequency separation required between any operating frequencies assigned to the nodes represented by the index pair.

An additional consideration must be taken into account in ordering nodes from most-to-least constrained. Each node may need a variable number of frequencies; it is possible that a node with a high number of requirements may have a high number of 
frequency separation constraints, as well. Making an assignment to such a node is doubly difficult in that prior assignments made will restrict frequencies available to this node, and more frequencies are required. The number of requirements and restrictions affecting a node should both be considered in order to assign frequencies first to those nodes anticipated to be the most difficult to accommodate. Achieving a maximally constrained ordering will therefore require examining the frequency separation matrix as well as the number of frequencies required by each node.

For each row of the frequency separation matrix, representing a given node, frequency separations are summed to assess the relative magnitude of constraints affecting each node. Nodes are sorted in descending order on the magnitude of their frequency separation constraint sums. Next, the number of such constraints is counted for each node; nodes are re-sorted again in descending order on the number of their constraints. The number of frequencies required for each node is the basis of the final sort, again in descending order. The machine index representing each node is referenced to the final ordering obtained.

As an alternative to ordering nodes by assignment difficulty, nodes may be ordered so that each frequency assignment to a given node preserves maximum flexibility for future assignments. ${ }^{31,38}$

Since machines represent nodes and frequencies assigned to nodes are represented by scheduled times of operations on machines, an ordering of nodes requiring the least additional spectrum to make an assignment of a frequency would tend to produce a schedule requiring the least additional time commitment for scheduling each operation within each job, and hence would tend to minimize frequency span and makespan respectively.

Nodes are first ordered by the number of constraints that their frequencies must obey; ordering is ascending, from lowest to highest number of applicable constraints. This ordering is checked for ties among nodes; nodes with the same number of constraints are ordered by the sum of their required frequency separations, in ascending order. In a sense, the ordering at the conclusion of this part of the process is opposite to that derived with the maximally constrained strategy; nodes are ordered by increasing constraint complexity. The procedure does not take into account the number of frequencies required per node. The object is a leastspectrum commitment ordering, in that making an assignment to each node in turn (i.e., scheduling each operation within a single job) commits the least additional frequency (time) to the total span of the frequency assignment (schedule). Therefore, the total number of requirements connected with any given node is not a consideration; the goal is to determine an ordering that permits completion of one assignment to each node in turn while retaining maximum flexibility for future assignments.

At the conclusion of the first stage of the least spectrum commitment ordering process, nodes are ordered from least constrained to most constrained. The second stage begins with the node at the top of the current list and searches the remainder of the list for the node requiring least frequency separation from the previous node, as this node would require the least additional spectrum commitment to be made in an assignment. This node becomes the second in the ordering. The remainder of the list is then searched for the node requiring the least frequency separation from those previously selected. This process is repeated until all nodes are listed; each additional node represents that which requires the commitment of the least additional spectrum as required by frequency separation constraints. Ordering nodes from least constrained to most constrained in the first stage of the process increases the chances of finding the least spectrum commitment-candidate node early in the list search, reducing the number of adjustments to the first stage ordering.

The Minimum Resource Model is more combinatorially complex than the Minimum Makespan Model. An efficient solution procedure explores only those solutions likely to produce the types of solutions sought - those which use the minimum number of machines necessary to achieve a successful schedule, within the minimum number of available machines required. It is clearly beneficial to use each individual machine for as many different tasks as possible. This will minimize the total number of machines which must be actively used ${ }^{31,34}$.

In the Minimum Resource Model, machines represent discrete frequency intervals; each job consists of assigning the required number of frequencies to a given node. Separations required between operating frequencies at different nodes, and at the same node, are implemented as restrictions on which machines may be used to perform operations. If no frequency separation is required between operating frequencies of 
a particular set of nodes, operations representing the assignment of a frequency to this set of nodes may be scheduled on the same machine.

The set of all operations associated with all jobs may be partitioned into groups of operations which may be performed on the same machine. While this process does not produce an assignment, it determines the minimum number of resources (machines) that are required to achieve one, and will achieve the primary objective of the Minimum Resource Model. Once a partition is constructed, all members of each partition may be scheduled on a machine, meeting all constraints and minimizing the total number of available (both active and idle) resources required.

Constructing a set partition is a relatively simple procedure. Consider a frequency separation matrix, and a list of requirements for all nodes, as described for the Minimum Makespan Model. Clearly, any two requirements for two different nodes requiring zero frequency separation may be placed in the same partition. Thus, any two operations, associated with two different jobs, requiring zero machine displacement, should be placed in the same partition. All operations of all jobs are examined in turn for possible membership in a partition; membership in a partition of operations associated with jobs will require that each member operation needs zero machine displacement from all other member operations. Each operation of each job will be considered for membership in an existing partition; while membership may be possible in a number of existing partitions, the operation will be placed as a member in the first such partition encountered, to speed the partitioning process. If no existing partition accommodates the operation being examined, a new partition is created which contains the operation as its first (and possibly, only) member.

While specific machines to which the operations will be assigned have not been determined, the number of partitions created yields the number of active machines that will need to be used. A feasibility check may now be performed to determine that at least this number of machines are available. If they are not, then the frequency assignment interpretation of this condition is that insufficient bandwidth exists to make a complete assignment satisfying all constraints. If sufficient resources are available, the next step in the solution process may be performed: determining a specific assignment of partitions to machines.
Developing a schedule of operations on the available machines is itself made easier by the set partitioning process. In determining a schedule, possible machines for entire partitions may be considered, which is less complex and lengthy than examining all possible machines that might be used for each individual operation of each and every job. However, there are a number of machines available to perform the operations associated with each given partition. A variety of possible solutions exists that are of equal quality with respect to the objective of minimizing the use of active resources.

However, there is a secondary objective in the Minimum Resource Model. By scheduling partitions of operations on machines that have as little relative displacement as possible, the objective of minimizing the number of both active and idle resources that are required for a solution can be achieved secondarily, minimizing the frequency span for the assignment.

The process of assigning partitions to machines is analogous to the Least Spectrum Commitment ordering strategy for the Minimum Makespan Model. Each new partition will be selected for scheduling to result in the minimum increment in total machine index, resulting in minimization of the total number of active and idle machines needed to complete the schedule.

The first partition scheduled is that with the maximum number of member operations. Remaining partitions will each be examined to determine which candidate should be scheduled next. Candidates are assessed on the basis of the new maximum machine index that would be required if that candidate were to be scheduled, by examining each candidate partition's required machine displacement from each of the partitions already scheduled. While all member operations of a candidate partition require zero machine displacement with respect to one another, members may require different displacements from members of other partitions. Thus, assessing machine displacement required between two partitions requires that machine displacement between all possible pairs of their respective members be assessed; the displacement between partitions is the maximum required between any two members of the two respective partitions. The maximum new machine index that is required to schedule a given partition is obtained by calculating required displacement between the candidate partition and each previously scheduled partition, and adding that displacement to the machine index corresponding to the 
scheduled partition. This process is followed for each unscheduled partition; the partition requiring minimum commitment of additional machine resources (both active and idle) is selected - i.e, the partition requiring the lowest new machine index is selected.

\section{$\underline{4.0 \text { Results }}$}

Computer codes were written to implement the Minimum Makespan Model, Minimum Resource Model and solution procedures described in preceding sections. Models and solution procedures were applied to several test cases. Test case 1 is a 5-node example, developed to verify performance of the models for problems allowing nodes to require different numbers of operating frequencies. Test case 2 is adapted from an 8-node example used by Hale ${ }^{18}$ to illustrate the difference between minimum span frequency assignment and minimum order frequency assignment, and is used for verification purposes here. Test case 3 is a highly constrained, 16-node problem of significant difficulty presented by Box ${ }^{4}$. This case was selected to provide a substantial challenge to the models and solution procedures presented here; ten iterative applications of an alternative heuristic procedure ${ }^{4}$ were required to complete the assignment. Test cases are summarized in Tables 1, 2, and 3.

\subsection{Results of the Minimum Makespan Model}

The Minimum Makespan Model, with the two ordering strategies defined in section 3.0, was applied to test cases 1, 2, and 3 . Results for each case are summarized in Tables 4, 5 and 6 which present resulting schedules and corresponding frequency assignments.

For test case 1, the Minimum Makespan Model achieves frequency assignments with spans of 180.0 and 190.0 for the Maximally Constrained and Least Spectrum Commitment ordering strategies respectively; the makespan of 180.0 is optimal for this example (as may be demonstrated through exhaustive enumeration). In Test Case 1, the Maximally Constrained ordering strategy performed better than the Least Spectrum Commitment strategy. Since the Maximally Constrained ordering strategy takes into account the number of frequencies required for each node, it is expected that this strategy would achieve superior performance for problems with variable requirements for frequency assignments at each node.

For test case 2, the Minimum Makespan Model performed identically for both ordering strategies, achieving frequency assignments of the optimum makespan of 40.0. This example demonstrates that the schedule construction process does produce a minimum makespan schedule and a minimum span frequency assignment subject to the ordering imposed. For a relatively minimally constrained problem such as test case 2, a variety of minimum span assignments are possible; two such assignments were found with the Maximally Constrained and Least Spectrum Commitment ordering strategies.

Test case 3 represents a problem of substantial difficulty. Consisting of 16 nodes, each requiring a single frequency assignment, the problem is highly constrained (See Table 3 ). This problem was originally utilized to demonstrate a heuristic procedure for simultaneously attempting frequency assignment and assessing assignment difficulty. The procedures utilized required 10 attempts to achieve a feasible frequency assignment for all 16 sites; the first two attempts yielded an assignment to 13 of the 16 sites ( 3 unassigned); the third through eighth attempts resulted in assignments to 14 of the 16 sites ( 2 unassigned); the ninth attempt assigned 15 of 16 sites $^{4}$.

For this test case, the Least Spectrum Commitment Ordering Strategy resulted in a near-complete assignment; two nodes did not receive frequency assignments. The Maximally Constrained ordering strategy resulted in an incomplete assignment; eight nodes did not receive assigned frequencies. While a complete assignment is not achieved for this example, the results are encouraging. Recall that the procedure presented in [4] required three to eight applications to achieve assignment of frequencies to 14 of 16 nodes; a comparable result is achieved by the Minimum Makespan Model with Least Spectrum Commitment ordering in a single application. This suggests that Least Spectrum Commitment Ordering is, at least, a useful initial ordering strategy for very highly constrained problems.

The results achieved for test case 3 raise some question regarding the desirability of utilizing the Minimum Makespan Model for highly constrained problems, since a complete solution was not found. A complete feasible solution exists ${ }^{4}$; since the node ordering utilized in the successful solution found in [4] differs markedly from both the Maximally Constrained and Least Spectrum Commitment orderings for this test case, it is reasonable to conjecture that neither ordering 
strategy is best for test case 3. However, a potentially productive coupling exists between an iterative procedure to assess assignment difficulty, such as that presented in [4], and the Minimum Makespan Model. Since the Minimum Makespan Model conserves spectrum, it is possible that an iterative assignment process could conclude earlier, using less frequency span, if the Minimum Makespan Model was incorporated as a method of determining an assignment once an ordering is determined.

Test cases examined demonstrate the impact of node ordering on schedule makespan and frequency span that may be achieved with the Minimum Makespan Model. The two ordering strategies specifically developed for the model, Maximally Constrained ordering and Least Spectrum Commitment ordering demonstrate specific performance tendencies in test cases 1 through 3 . It is expected that the Maximally Constrained ordering strategy would exhibit better performance for problems where nodes require a variable number of assigned frequencies, since this strategy takes this factor into account. However, this strategy does not order nodes in relationship to one another; the strategy merely ranks nodes from most to least constrained. The Least Spectrum Commitment ordering strategy may be the strategy of choice for highly constrained problems, as nodes are ordered in terms of their relationship to one another. As both strategies are easily implemented, the best approach may be to attempt both strategies for a given problem, and adopt the best assignment found. As was demonstrated with test case 2, it is possible for both strategies to perform equally well with respect to makespan (frequency span) while determining alternate assignments. From test case 3, Least Spectrum Commitment ordering appears to be more effective for highly constrained problems. It is apparent that scheduling the most highly constrained operation first, followed by operations successively less constrained, as is done with Maximally Constrained ordering, exhausts the time (frequency) span available relatively early in the process; the first eight nodes in the Maximally Constrained ordering receive assignments, at which point the available time (frequency) is exhausted. Least Spectrum Commitment ordering permits the scheduling process to continue further than does the other strategy, by preserving a larger portion of the available time span for scheduling additional operations.

\section{4,2 Results of the Minimum Resource Model}

The Minimum Resource Model was applied to Test
Cases 1, 2, and 3. Results for each test case are summarized in Tables 7, 8, and 9, which present resulting schedules and corresponding frequency assignments.

For test case 1, the Minimum Resource Model achieved an assignment utilizing $\mathbf{1 1}$ active frequencies, which is the minimum number achievable for this test case consistent with satisfying all frequency separation constraints.

For test case 2, the Minimum Resource Model found an assignment with the demonstrated minimum number ${ }^{18}$ of required frequencies for the assignment. The Minimum Resource Model thus does achieve minimum order frequency assignment. Since this test problem is not highly constrained, and each subset of the set partitioning of sites contains more than 1 member (Partition 1 and Partition 2 contain 4 members each, respectively), a redundant solution is also possible, by interchanging the frequencies associated with each partition. For more highly constrained problems this may not be the case.

For test case 3, the Minimum Resource Model is able to achieve a partial assignment of frequencies to 15 of 16 sites, using a total of 13 frequencies of the 21 available (14 are determined to be necessary through set partitioning.) The ratio of required number of frequencies, 14 , to the total number of requirements, 16 , is high; this is one indication of the high degree of difficulty of determining an assignment for test case 3. While a complete assignment was not achieved, the results are very encouraging. Recall that the procedure utilized in [4] required nine applications before achieving an assignment to 15 of 16 sites for this test case; the Minimum Resource Model achieved similar results with a single application, suggesting that the Minimum Resource Model may be a viable approach to highly constrained problems.

While a feasible assignment was not achieved for test case 3, the Minimum Resource Model might be coupled with an assignment ordering strategy, such as that presented in [4], with productive results. Since the Minimum Resource Model minimizes the number of discrete frequencies utilized to achieve an assignment, it is conceivable that its use as an assignment procedure in an iterative process like that of [4] might result in earlier solution of the assignment problem.

\subsection{Comparison of Model Results}


The Minimum Makespan and Minimum Resource Models are designed to represent two alternative views of spectrum conservation. The Minimum Makespan Model achieves frequency assignment using a minimum range (lowest to highest) of frequencies. The Minimum Resource Model achieves frequency assignment using the minimum number of frequencies possible. A tradeoff between the two models may be exhibited, in that a wider range of frequencies may be required to achieve assignment with a minimum number of frequencies; conversely, a larger number of frequencies may be required to achieve an assignment within a minimum range of frequencies. It is also expected that one model may exhibit better performance than the other for specific types of problems.

For test case 1, the Minimum Makespan Model achieved assignments with total frequency spans of 180.0 and 190.0, utilizing 13 and 14 frequencies, for the Maximally Constrained and Least Spectrum Commitment ordering strategies respectively. The Minimum Resource model achieved an assignment for test case 1 with a total frequency span of 220.0 , utilizing 11 frequencies. Thus, for Test Case 1 , a tradeoff of exactly the type expected is observed - i.e., lower frequency span, with more frequencies used, versus high frequency span, with fewer frequencies used.

For test case 2, the Minimum Makespan Model achieved a frequency assignment with a total span of 40 , utilizing 3 frequencies, for all ordering strategies. The Minimum Resource Model achieved a frequency assignment, with a total span of 40 , utilizing 2 frequencies. In this case, the tradeoff expected between the two models is not exhibited; both models achieve the same frequency span, but the Minimum Resource Model requires one fewer frequency. It cannot be stated definitively that the Minimum Resource Model achieves superior performance, however; the usefulness of conserving the frequency not utilized in the assignment, which is located between two other frequencies, depends upon whether the frequency may truly be utilized for additional requirements. As a general principle, however, it would be beneficial to conserve frequencies if doing so requires no increase in frequency span over that achieved with a minimum span assignment.

For test case 3, the most difficult of the test cases, the Minimum Resource Model can be said to demonstrate superior performance over the Minimum Makespan Model, in that an assignment of 15 of 16 required frequencies is accomplished, as opposed to 14 of 16 - the best achieved with the Minimum Makespan Model. It is interesting to note that the performance of the Minimum Resource Model and Minimum Makespan Model with Least Spectrum Commitment ordering are nearly equal in quality; this is not surprising, because the two approaches are somewhat related. The Minimum Resource Model employs set partitioning to group together requirements that may be satisfied by the same frequency; the Least Spectrum Commitment ordering strategy will tend to place requirements that may share the same frequency in adjacent "slots" in the ordering. The total frequency span utilized for the Minimum Resource assignment is 525.0; that achieved with the Minimum Makespan Model is $\mathbf{5 0 0 . 0}$. However, since an additional node receives an assignment via the Minimum Resource Model, it is not surprising that a greater span is utilized in the partial assignment achieved. A direct comparison of the frequency spans achieved by each model is not truly possible for this example.

\subsection{Summary and Conclusions}

Alternative definitions of spectrum conservation exist. Spectrum-conserving frequency assignment may be defined in one of at least two ways. Minimum span frequency assignment minimizes the range of frequencies that must be used to meet a given set of demands. This objective is desired in portions of the spectrum allocated to two or more incompatible services, because it conserves the largest continuous portion of the spectrum for future assignment. Minimum order frequency assignment meets a specific set of demands with the minimum number of frequencies. This objective is suitable in portions of the spectrum allocated to just one service, where it is desirable to preserve the maximum number of channels for future assignment. These alternate objectives are conflicting. To achieve an assignment with the minimum number of frequencies, it may be necessary to use a wider range of frequencies; to achieve an assignment within a minimum frequency span, it may be necessary to use a higher number of frequencies. The second consideration in development of a general frequency assignment procedure is the computational complexity of the frequency assignment problem. Regardless of the objective, such problems are NP-hard or NP-complete. A variety of solutions are possible for any given problem; the task of enumerating all possible solutions in search of one - or more - optima is not practically possible. 
A general approach to the frequency assignment problem has been developed that employs $\mathrm{N}$-job, M-machine scheduling models to achieve spectrum-conserving frequency assignments. Two models were formulated, to correspond to two alternative and conflicting views of spectrum conservation.

The Minimum Makespan Model was developed to achieve minimum span frequency assignment. Nodes requiring frequencies are modeled as machines; assigned frequencies are modeled as assigned times within a schedule; the assignment of frequencies to requirements of each site is modeled as a series of jobs composed of operations that must be performed on different machines. While job processing order does not impact makespan, machine order (node order) does. Two alternative ordering strategies were developed that rely on insights gained from previous research on the frequency assignment problem. The Maximally Constrained Ordering Strategy seeks to determine the "most difficult" assignments first, on the assumption that requirements of lesser difficulty will be flexible enough to be assigned successfully later. The Least Spectrum Commitment Ordering embodies a different philosophy; sites are ordered so as to commit the least additional spectrum with each successive assignment, on the assumption that maximal flexibility is retained for assignments not yet made.

The Minimum Resource Model was developed to determine minimum order frequency assignments. Discrete frequencies are modeled as machines; each node's set of required frequency assignments is modeled as a single job with the required number of operations. Set partitioning is employed to determine the minimum number of frequencies necessary to provide the assignment; the model seeks to provide this number of frequencies within the minimum span consistent with satisfying frequency separation constraints.

As demonstrated with a variety of test cases, the models and solution procedures developed achieve their intended purposes. As might be expected, performance varies with the specific problem addressed. Both models can be expected to perform well for moderately sized problems of moderate complexity when the number of requirements at all nodes is uniform. For problems in which the number of frequencies required by each node is variable, both the Minimum Makespan Model with Maximally Constrained ordering and the Minimum
Resource Model tend to yield good performance. For highly constrained problems, both the Minimum Makespan Model with Least Spectrum Commitment Ordering and the Minimum Resource Model appear to be viable alternatives. It is encouraging that both a minimum span and minimum order approach seem to be available for most types of problems that might be expected to be encountered, suggesting that a viable scheduling approach to frequency assignment exists for portions of the spectrum facing different demands.

Further efforts in general approaches to solution of the frequency assignment problem are needed. For instance, this research has explored rather than resolved the question of ordering assignment requirements in the frequency assignment problem. As demonstrated via performance in test cases, this research has verified the impact of requirements ordering on the success or failure of a frequency assignment procedure. It is reasonable to conjecture that no one requirements ordering strategy will perform successfully for all problems, and that an iterative approach to requirements ordering may be needed for very highly constrained problems.

Further investigation is also required into the relationship between minimum span frequency assignment and minimum order frequency assignment. While the two objectives conflict, there remains the possibility that the most beneficial approach to spectrum conservation lies in the direction of a mulliobjective treatment of the frequency assignment problem.

The frequency assignment problem is a problem in resource allocation. The communications frequency spectrum is a limited resource, facing increasing demands as new communication applications and services are developed. As is true for any limited resource, conservation and efficient use of the frequency spectrum are necessary to ensure that both present and future demands on the resource may be met. Treatment of the frequency assignment problem as a scheduling problem provides a viable general approach to achieving spectrum-conserving frequency assignment. 


\section{References}

1 Anderson, Lewis G. "A Simulation Study of Some Dynamic Channel Assignment Algorithms in a High Capacity Mobile Telecommunications System," IEEE Transactions on Vehicular Technology, Vol. VT-22, No. 4, November, 1973, pp. 210-217.

2 Arnaud, Jean-Francois. "Frequency Planning for Broadcast Service in Europe," Proceedings of the IEEE, Vol. 68, No. 12, December 1980, pp.15151522 .

3 Baker, Kenneth R., Introduction to Sequencing and Scheduling, John Wiley and Sons, Inc., New York, 1974.

4 Box, Frank. "A Heuristic Technique for Assigning Frequencies to Mobile Radio Nets," IEEE Transactions on Vehicular Technology, Vol. VT-27, No. 2, May 1978, pp.57-64.

5 Brelaz, Daniel. "New Methods to Color the Vertices of a Graph," Communications of the ACM, Vol. 22, No.4, April 1979, pp. 251-256.

6 Cameron, Scott H. "The Solution of the GraphColoring Problem as a Set-Covering Problem," IEEE Transactions on Electromagnetic Compatibility, Vol. EMC-19, No. 3, August 1977, pp. 320-322.

7 Catlin, Paul A. "A Bound on the Chromatic Number of a Graph," Discrete Mathematics 22 (1978), NorthHolland Publishing Company, pp. 81-83.

8 Catlin, Paul A. "Another Bound on the Chromatic Number of a Graph," Discrete Mathematics 24 (1978), North-Holland Publishing Company, pp. 1-6.

9 Corneil, D. G., and B. Graham. "An Algorithm for Determining the Chromatic Number of a Graph," SIAM J. Comput., Vol. 2, No. 4,December 1973, pp.311-318.

10 Cox, D. C., and D. O. Reudink. "Dynamic Channel Assignment in Two-Dimensional Large-Scale Mobile Radio Systems," The Bell System Technical Journal, Vol. 51, No. 7, September 1972, pp.1611-1629.
11 Cox, D. C., and D. O. Reudink. "Increasing Channel Occupancy in Large-Scale Mobile Radio Systems: Dynamic Channel Reassignment," IEEE Transactions on Vehicular Technology, Vol. VT-22, No. 4,November 1973, pp. 218-222.

12 Dash, M. J., and S. R. Green. "Parameter Sensitivity Analysis: An Approach Used in the Investigation of Frequency Assignment Problems," Proceedings of the Conference on Electromagnetic Compatibility,Guilford, England, pp. 55-64, April 1978.

13 Fang, R. J. F., and W. A. Sandrin. "Carrier frequency assignment for nonlinear repeaters," COMSAT Technical Review, Vol.7, No. 1, Spring 1977, pp.27. 245.

14 Freeman, Ernest R. "An Approach to Frequency Assignment," IEEE Transactions on Electromagnetic Compatibility, Vol. EMC-8, No. 2, June 1966, pp. 9096.

15 Garey, Michael R., and Davis S. Johnson. Computers and Intractability: A Guide to the Theory of NP-Completeness, W. H. Freeman and Co., San Francisco, 1979, Chapter 5.

16 Garey, M. R., and D. S. Johnson. "The Complexity of Near-Optimal Graph Coloring," Journal of the ACM, Vol. 23, No. 1, January 1976, pp.43-49.

17 Graves, Stephen C., "A Review of Production Scheduling", Operations Research, Vol. 29, No. 4, July-August 1984, pp. 646-675.

18 Hale, William K. "Frequency Assignment: Theory and Applications," Proceedings of the IEEE, Vol. 68, No. 12, December, 1980, pp. 1497-1514.

19 Ignizio, James P. "A Generalized Goal Programming Approach to the Minimal Interference, Multicriteria Nx1 Scheduling Problem," IIE Transactions, Vol. 16, No. 4, December, 1984, pp. 316-322.

20 Ito, Yasuhiko; Mizuno, Toshio, and Takuro Muratani. "Effective Utilization of Geostationary Orbit Through Optimization," IEEE Transactions on Communications, Vol. COM-27, No. 10, October, 1979, pp.1551-1558. 
21 Kalnin'sh, A. A. "The Coloring of Graphs in a Linear Number of Steps," Cybernetics, Vol. 7, No. 4, pp. 699-700, July-August, 1971.

22 Lageweg, B.J., Lenstra, J.K., and A.H.G. Rinnooy Kan, "Job-Shop Scheduling by Implicit Enumeration", Management Science, Vol. 24, No.4, December 1977, pp. 441-450.

23 Mathur, Kamlesh; Salkin, Harvey M.; Nishimura, Koichi, and Susumu Morito. "The Design of an Interactive Computer Software System for the Frequency-Assignment Problem,"IEEETransactions on Electromagnetic Compatibility, Vol. EMC-26, No. 4, November, 1984, pp. 207-212.

24 Mathur, Kamlesh; Salkin, Harvey M., Nishimüra, Koichi,and Susumu Morito. "Applications of Integer Programming in Radio Frequency Management,"Management Science, Vol. 31, No. 7, July 1985,pp. 829-839.

25 Maxemchuk, N. F., and L. Schiff. "Third-Order Intermodulation Interference - Bounds and Interference-Free Channel Assignment," IEEE Transactions on Communications, Vol. COM-25, No.9, September 1977, pp. 1041-1046.

26 McDiarmid, C. "Determining the Chromatic Number of a Graph," SIAM J. Comput., Vol. 8, No. 1, February 1979, pp. 1-14.

27 Mitchem, John. "On various algorithms for estimating the chromatic number of a graph," The Computer Journal, Vol. 19, No. 3, May 1976, pp. 182-183.

28 Mizuike, Takeshi; Ito, Yasuhiko, and Takuro Muratani. "Optimization of Frequency Assignment," IEEE International Conference on Communications,Amsterdam, The Netherlands, May, 1984.

29 Morito, Susumu; Salkin, Harvey M., and Kamlesh Mathur. "Computational Experience With a Dual Backtrack Algorithm for Identifying Frequencies Likely to Create Intermodulation Problems," IEEE Transactions on Electromagnetic Compatibility, Vol. EMC-23, No. 1, February 1981, pp. 32-36.
30 Okinaka, Hideo; Yasuda, Yukata, and Yasuo Hirata."Intermodulation Interference-Minimum Frequency Assignment for Satellite SCPC Systems," IEEE Transactions on Communications, Vol.COM32, No. 4, April 1984, pp.462-468.

31 Randall-Brown, J. "Chromatic Scheduling and the Chromatic Number Problem," Management Science, Vol.19, No. 4, December, Part I, 1972, pp.456-463.

32 Sivyer, G. R., and S. R. Inge. "A Practical A Priori Method of Frequency Assignment for Multiple Transmitters and Sites which Minimises the Total Radio Spectrum Required," Broadcasting Corporation of New Zealand.

33 Vuong, X. T.; Ozmizrak, F. N.; Birta, L. G., and K. D. Nguyen. "Investigation of Practical Strategies for Solving the One-Level Carrier Frequency Assignment Problem," GLOBECOM '87 Proceedings, pp. 2165-2169.

34 Wang, Chung C. "An Algorithm for the Chromatic Number of a Graph," Journal of the ACM, Vol.21, No. 3, July 1974, pp.385-391.

35 Weiss, Hans J. "Relating to the Efficiency of Utilization of the Geostationary Orbit/Spectrum in the Fixed Satellite Service," Proceedings of the IEEE, Vol. 68, No. 12, December, 1980, pp. 1484 1496.

36 Welsh, D. J. A., and M. B. Powell. "An upper bound for the chromatic number of a graph and its application to timetabling problems," Computer Journal, Vol.10, 1967, pp.85-86.

37 Wood, D. C. "A technique for colouring a graph applicable to large scale timetabling problems," Comput. Journal, Vol. 12, 1968, pp. 317-319.

38 Zoellner, J. Arthur. "Frequency Assignment Games and Strategies," IEEE Transactions on Electromagnetic Compatibility, Vol. EMC-15, No. 4, November 1973, pp. 191-196.

39 Zoellner, J. Arthur, and C. Lyle Beall. "A Breakthrough in Spectrum Conserving Frequency Assignment Technology," IEEE Transactions on Electromagnetic Compatibility, Vol. EMC-19, No.3, August 1977, pp. 313-319. 
Table 1(a):

Frequency Separation Matrix, Test Case 1

\begin{tabular}{||c|c|c|c|c|c||}
\hline Node & 1 & 2 & 3 & 4 & 5 \\
\hline 1 & 0.0 & 0.0 & 10.0 & 0.0 & 20.0 \\
\hline 2 & 0.0 & 0.0 & 10.0 & 10.0 & 10.0 \\
\hline 3 & 10.0 & 10.0 & 0.0 & 10.0 & 10.0 \\
\hline 4 & 0.0 & 10.0 & 10.0 & 0.0 & 10.0 \\
\hline 5 & 20.0 & 10.0 & 10.0 & 10.0 & 0.0 \\
\hline
\end{tabular}

Table 1(b):

Problem Requirements, Test Case 1

\begin{tabular}{||c|c|c||}
\hline Node & $\begin{array}{l}\text { \# Frequencies } \\
\text { Required }\end{array}$ & $\begin{array}{l}\text { Bandwidth } \\
\text { Required }\end{array}$ \\
\hline 1 & 4 & 20.0 \\
\hline 2 & 3 & 20.0 \\
\hline 3 & 2 & 20.0 \\
\hline 4 & 1 & 20.0 \\
\hline 5 & 5 & 20.0 \\
\hline
\end{tabular}

Minimum Frequency: $\quad 600.0$

Maximum Frequency: $\quad 800.0$
Total Bandwidth: $\quad 200.0$

Normalization Factor: 10.0 
Table 2(a):

Frequency Separation Matrix, Test Case 2

\begin{tabular}{||c|c|c|c|c|c|c|c|c||}
\hline Node & 1 & 2 & 3 & 4 & 5 & 6 & 7 & 8 \\
\hline 1 & 0.0 & 20.0 & 0.0 & 0.0 & 0.0 & 0.0 & 0.0 & 0.0 \\
\hline 2 & 20.0 & 0.0 & 0.0 & 0.0 & 0.0 & 0.0 & 0.0 & 0.0 \\
\hline 3 & 0.0 & 0.0 & 0.0 & 20.0 & 0.0 & 0.0 & 0.0 & 0.0 \\
\hline 4 & 0.0 & 0.0 & 20.0 & 0.0 & 10.0 & 0.0 & 0.0 & 0.0 \\
\hline 5 & 0.0 & 0.0 & 0.0 & 10.0 & 0.0 & 10.0 & 0.0 & 0.0 \\
\hline 6 & 0.0 & 0.0 & 0.0 & 0.0 & 10.0 & 0.0 & 0.0 & 0.0 \\
\hline 7 & 0.0 & 0.0 & 0.0 & 0.0 & 0.0 & 0.0 & 0.0 & 10.0 \\
\hline 8 & 0.0 & 0.0 & 0.0 & 0.0 & 0.0 & 0.0 & 10.0 & 0.0 \\
\hline
\end{tabular}

Table 2(b):

Problem Requirements, Test Case 2

\begin{tabular}{||c|c|c||}
\hline Node & $\begin{array}{l}\text { \# Frequencies } \\
\text { Required }\end{array}$ & $\begin{array}{l}\text { Bandwidth } \\
\text { Required }\end{array}$ \\
\hline 1 & 1 & 20.0 \\
\hline 2 & 1 & 20.0 \\
\hline 3 & 1 & 20.0 \\
\hline 4 & 1 & 20.0 \\
\hline 5 & 1 & 20.0 \\
\hline 6 & 1 & 20.0 \\
\hline 7 & 1 & 20.0 \\
\hline 8 & 1 & 20.0 \\
\hline
\end{tabular}

Minimum Frequency: $\quad 1000.0$ Maximum Frequency: 1160.0
Total Bandwidth: $\quad 160.0$

Normalization Factor: 10.0 
Table 3(a):

Frequency Separation Matrix, Test Case 3

\begin{tabular}{|l|l|l|l|l|l|l|l|l|l|l|l|l|l|l|l|}
\hline 0 & 75 & 50 & 0 & 50 & 50 & 50 & 25 & 50 & 50 & 50 & 50 & 25 & 25 & 25 & 0 \\
\hline 75 & 0 & 50 & 50 & 50 & 50 & 50 & 25 & 50 & 50 & 50 & 50 & 25 & 25 & 25 & 0 \\
\hline 50 & 50 & 0 & 75 & 75 & 75 & 75 & 50 & 50 & 50 & 50 & 50 & 25 & 25 & 25 & 25 \\
\hline 50 & 50 & 75 & 0 & 75 & 75 & 75 & 50 & 50 & 50 & 50 & 50 & 25 & 25 & 25 & 25 \\
\hline 50 & 50 & 75 & 75 & 0 & 75 & 75 & 50 & 50 & 50 & 50 & 50 & 25 & 25 & 25 & 25 \\
\hline 50 & 50 & 75 & 75 & 75 & 0 & 75 & 50 & 50 & 50 & 50 & 50 & 25 & 25 & 25 & 25 \\
\hline 50 & 50 & 75 & 75 & 75 & 75 & 0 & 50 & 50 & 50 & 50 & 50 & 25 & 25 & 25 & 25 \\
\hline 25 & 25 & 50 & 50 & 50 & 50 & 50 & 0 & 25 & 25 & 50 & 50 & 0 & 25 & 25 & 25 \\
\hline 50 & 50 & 50 & 50 & 50 & 50 & 50 & 25 & 0 & 75 & 50 & 50 & 50 & 50 & 50 & 25 \\
\hline 50 & 50 & 50 & 50 & 50 & 50 & 50 & 25 & 75 & 0 & 50 & 50 & 50 & 50 & 50 & 25 \\
\hline 50 & 50 & 50 & 50 & 50 & 50 & 50 & 50 & 50 & 50 & 0 & 50 & 50 & 50 & 50 & 50 \\
\hline 50 & 50 & 50 & 50 & 50 & 50 & 50 & 50 & 50 & 50 & 50 & 0 & 50 & 50 & 50 & 50 \\
\hline 25 & 25 & 25 & 25 & 25 & 25 & 25 & 0 & 50 & 50 & 50 & 50 & 0 & 50 & 50 & 25 \\
\hline 25 & 25 & 25 & 25 & 25 & 25 & 25 & 25 & 50 & 50 & 50 & 50 & 50 & 0 & 75 & 50 \\
\hline 25 & 25 & 25 & 25 & 25 & 25 & 25 & 25 & 50 & 50 & 50 & 50 & 50 & 75 & 0 & 50 \\
\hline 0 & 0 & 25 & 25 & 25 & 25 & 25 & 25 & 25 & 25 & 50 & 50 & 25 & 50 & 50 & 0 \\
\hline \hline
\end{tabular}


Table 3(b):

Problem Requirements, Test Case 3

\begin{tabular}{||c|c|c||}
\hline Node & $\begin{array}{l}\text { \# Frequencies } \\
\text { Required }\end{array}$ & $\begin{array}{l}\text { Bandwidth } \\
\text { Required }\end{array}$ \\
\hline 1 & 1 & 25.0 \\
\hline 2 & 1 & 25.0 \\
\hline 3 & 1 & 25.0 \\
\hline 4 & 1 & 25.0 \\
\hline 5 & 1 & 25.0 \\
\hline 6 & 1 & 25.0 \\
\hline 7 & 1 & 25.0 \\
\hline 8 & 1 & 25.0 \\
\hline 9 & 1 & 25.0 \\
\hline 10 & 1 & 25.0 \\
\hline 11 & 1 & 25.0 \\
\hline 12 & 1 & 25.0 \\
\hline 13 & 1 & 25.0 \\
\hline 14 & 1 & 25.0 \\
\hline 15 & 1 & 25.0 \\
\hline 16 & 1 & 25.0 \\
\hline \hline
\end{tabular}

Minimum Frequency: $\quad 1000.0$

Maximum Frequency. $\quad 1525.0$
Total Bandwidth: $\quad 525.0$

Normalization Factor: 25.0 
Table 4: Results of Minimum Makespan Model, Test Case 1

\begin{tabular}{|c|c|c|c|c|}
\hline & $\begin{array}{l}\text { Start } \\
\text { Time }\end{array}$ & $\begin{array}{l}\text { Start } \\
\text { Time }\end{array}$ & Frequency & Frequency \\
\hline $\begin{array}{l}\text { (Node, } \\
\text { Requirement) }\end{array}$ & $\begin{array}{l}\text { Maximally } \\
\text { Constrained } \\
\text { Ordering }\end{array}$ & $\begin{array}{l}\text { Least } \\
\text { Spectrum } \\
\text { Commitment } \\
\text { Ordering }\end{array}$ & $\begin{array}{l}\text { Maximally } \\
\text { Constrained } \\
\text { Ordering }\end{array}$ & $\begin{array}{l}\text { Least Spectrum } \\
\text { Commitment } \\
\text { Ordering }\end{array}$ \\
\hline$(1,1)$ & 3 & 1 & $620-640$ & $600-620$ \\
\hline$(1,2)$ & 7 & 6 & $660-680$ & $650-670$ \\
\hline$(1,3)$ & 11 & 10 & $700-720$ & $690-710$ \\
\hline$(1,4)$ & 15 & 14 & $740-760$ & $730-750$ \\
\hline$(2,1)$ & 3 & 1 & $620-640$ & $600-620$ \\
\hline$(2,2)$ & 7 & 5 & $660-680$ & $640-660$ \\
\hline$(2,3)$ & 10 & 9 & $690-710$ & $680-700$ \\
\hline$(3,1)$ & 2 & 3 & $610-630$ & $620-640$ \\
\hline$(3,2)$ & 6 & 7 & $650-670$ & $660-680$ \\
\hline$(4,1)$ & 4 & 2 & $630-650$ & $610-630$ \\
\hline$(5,1)$ & 1 & 4 & $600-620$ & $630-650$ \\
\hline$(5,2)$ & 5 & 8 & $640-660$ & $670-690$ \\
\hline$(5,3)$ & 9 & 12 & $680-700$ & $710-730$ \\
\hline$(5,4)$ & 13 & 16 & $720-740$ & $750-770$ \\
\hline$(5,5)$ & 17 & 18 & $760-780$ & $770-790$ \\
\hline $\begin{array}{l}\text { Schedule/ } \\
\text { Frequency Span }\end{array}$ & 18 & 19 & 180 & 190 \\
\hline $\begin{array}{l}\text { \# Active } \\
\text { Frequencies }\end{array}$ & & & 13 & 14 \\
\hline
\end{tabular}


Table 5: Results of Minimum Makespan Model, Test Case 2

\begin{tabular}{||l|l|l|l|l||}
\hline & $\begin{array}{l}\text { Start } \\
\text { Time }\end{array}$ & $\begin{array}{l}\text { Start } \\
\text { Time }\end{array}$ & Frequency & Frequency \\
\hline $\begin{array}{l}\text { (Node, } \\
\text { Requirement) }\end{array}$ & $\begin{array}{l}\text { Maximally } \\
\text { Constrained } \\
\text { Ordering }\end{array}$ & $\begin{array}{l}\text { Least } \\
\text { Spectrum } \\
\text { Commitment } \\
\text { Ordering }\end{array}$ & $\begin{array}{l}\text { Maximally } \\
\text { Constrained } \\
\text { Ordering }\end{array}$ & $\begin{array}{l}\text { Least Spectrum } \\
\text { Commitment } \\
\text { Ordering }\end{array}$ \\
\hline$(1,1)$ & 1 & 1 & $1000-1020$ & $1000-1020$ \\
\hline$(2,1)$ & 3 & 3 & $1020-1040$ & $1020-1040$ \\
\hline$(3,1)$ & 3 & 1 & $1020-1040$ & $1000-1020$ \\
\hline$(4,1)$ & 1 & 3 & $1000-1020$ & $1020-1040$ \\
\hline$(5,1)$ & 2 & 2 & $1010-1030$ & $1010-1030$ \\
\hline$(6,1)$ & 1 & 1 & $1000-1020$ & $1000-1020$ \\
\hline$(7,1)$ & 2 & 1 & $1000-1020$ & $1000-1020$ \\
\hline$(8,1)$ & & 2 & $1010-1030$ & $1010-1030$ \\
\hline $\begin{array}{l}\text { Schedule/ } \\
\text { Frequency Span }\end{array}$ & 1 & 40 & 40 \\
\hline $\begin{array}{l}\text { \# Active } \\
\text { Frequencies }\end{array}$ & & & 3 & 3 \\
\hline
\end{tabular}


Table 6: Results of Minimum Makespan Model, Test Case 3

\begin{tabular}{|c|c|c|c|c|}
\hline & $\begin{array}{l}\text { Start } \\
\text { Time }\end{array}$ & $\begin{array}{l}\text { Start } \\
\text { Time }\end{array}$ & Frequency & Frequency \\
\hline $\begin{array}{l}\text { (Node, } \\
\text { Requirement) }\end{array}$ & $\begin{array}{l}\text { Maximally } \\
\text { Constrained } \\
\text { Ordering }\end{array}$ & $\begin{array}{l}\text { Least } \\
\text { Spectrum } \\
\text { Commitment } \\
\text { Ordering } \\
\end{array}$ & $\begin{array}{l}\text { Maximally } \\
\text { Constrained } \\
\text { Ordering }\end{array}$ & $\begin{array}{l}\text { Least Spectrum } \\
\text { Commitment } \\
\text { Ordering }\end{array}$ \\
\hline$(1,1)$ & * & 4 & * & $1074-1100$ \\
\hline$(2,1)$ & $*$ & 1 & $*$ & $1000-1025$ \\
\hline$(3,1)$ & 1 & 6 & $1125-1150$ & $1125-1150$ \\
\hline$(4,1)$ & 9 & 9 & $1200-1225$ & $1200-1225$ \\
\hline$(5,1)$ & 12 & 20 & $1275-1300$ & $1475-1500$ \\
\hline$(6,1)$ & 15 & 13 & $1350-1375$ & $1300-1325$ \\
\hline$(7,1)$ & 18 & 17 & $1425-1500$ & $1400-1425$ \\
\hline$(8,1)$ & $*$ & 2 & $*$ & $1025-1050$ \\
\hline$(9,1)$ & 20 & 11 & $1475-1500$ & $1250-1275$ \\
\hline$(10,1)$ & * & 15 & * & $1350-1375$ \\
\hline$(11,1)$ & 1 & $*$ & $1000-1025$ & $*$ \\
\hline$(12,1)$ & 4 & * & $1075-1100$ & $*$ \\
\hline$(13,1)$ & * & 2 & * & $1025-1050$ \\
\hline$(14,1)$ & * & 5 & $*$ & $1100-1125$ \\
\hline$(15,1)$ & * & 8 & * & $1175-1200$ \\
\hline$(16,1)$ & * & 1 & $*$ & $1000-1025$ \\
\hline $\begin{array}{l}\text { Schedule/ } \\
\text { Frequency Span }\end{array}$ & 20 & 20 & 500 & 500 \\
\hline $\begin{array}{l}\text { \# Active } \\
\text { Frequencies }\end{array}$ & & & 8 & 12 \\
\hline
\end{tabular}


Table 7: Results of Minimum Resource Model, Test Case 1:

\begin{tabular}{|c|c|c|}
\hline $\begin{array}{l}\text { (Node, } \\
\text { Requirement) }\end{array}$ & Assigned Machine & $\begin{array}{l}\text { Assigned } \\
\text { Frequency }\end{array}$ \\
\hline$(1,1)$ & 1 & $600-620$ \\
\hline$(1,2)$ & 2 & $610-630$ \\
\hline$(1,3)$ & 3 & $620-640$ \\
\hline$(1,4)$ & 4 & $630-650$ \\
\hline$(2,1)$ & 1 & $600-620$ \\
\hline$(2,2)$ & 2 & $610-630$ \\
\hline$(2,3)$ & 3 & $620-640$ \\
\hline$(3,1)$ & 5 & $640-660$ \\
\hline$(3,2)$ & 6 & $650-670$ \\
\hline$(4,1)$ & 4 & $630-650$ \\
\hline$(5,1)$ & 7 & $660-680$ \\
\hline$(5,2)$ & 8 & $670-690$ \\
\hline$(5,3)$ & 9 & $680-700$ \\
\hline$(5,4)$ & 10 & $690-710$ \\
\hline$(5,5)$ & 11 & $700-720$ \\
\hline $\begin{array}{l}\text { Frequency } \\
\text { Span }\end{array}$ & & 220 \\
\hline $\begin{array}{l}\text { Total } \\
\text { Number Machines/ } \\
\text { Frequencies } \\
\text { Required } \\
\text { (Active/Idle) } \\
\end{array}$ & 20 & 20 \\
\hline \# Active Frequencies & 11 & 11 \\
\hline
\end{tabular}


Table 8: Results of Minimum Resource Model, Test Case 2:

\begin{tabular}{||c|c|c||}
\hline (Node, Requirement) & $\begin{array}{l}\text { Assigned } \\
\text { Machine }\end{array}$ & $\begin{array}{l}\text { Assigned } \\
\text { Frequency }\end{array}$ \\
\hline$(1,1)$ & 1 & $1000-1020$ \\
\hline$(2,1)$ & 3 & $1020-1040$ \\
\hline$(3,1)$ & 1 & $1000-1020$ \\
\hline$(4,1)$ & 3 & $1020-1040$ \\
\hline$(5,1)$ & 1 & $1000-1020$ \\
\hline$(6,1)$ & 3 & $1020-1040$ \\
\hline$(7,1)$ & 1 & $1000-1020$ \\
\hline$(8,1)$ & 3 & $1020-1040$ \\
\hline $\begin{array}{l}\text { Frequency } \\
\text { Span }\end{array}$ & 3 & 40 \\
\hline $\begin{array}{l}\text { Total } \\
\text { Number Machines/ } \\
\text { Frequencies Required } \\
\text { (Active/Idle) }\end{array}$ & 2 & 3 \\
\hline $\begin{array}{l}\text { \# Active } \\
\text { Machines/ } \\
\text { Frequencies }\end{array}$ & & 2 \\
\hline
\end{tabular}


Table 9: Results of Minimum Resource Model, Test Case 3

\begin{tabular}{|c|c|c|}
\hline (Node, Requirement) & $\begin{array}{l}\text { Assigned } \\
\text { Machine }\end{array}$ & $\begin{array}{l}\text { Assigned } \\
\text { Frequency }\end{array}$ \\
\hline$(1,1)$ & 1 & $1000-1025$ \\
\hline$(2,1)$ & 4 & $1075-1100$ \\
\hline$(3,1)$ & 6 & $1125-1150$ \\
\hline$(4,1)$ & 10 & $1225-1250$ \\
\hline$(5,1)$ & 13 & $1300-1325$ \\
\hline$(6,1)$ & 17 & $1400-1425$ \\
\hline$(7,1)$ & 21 & $1500-1525$ \\
\hline$(8,1)$ & 2 & $1025-1050$ \\
\hline$(9,1)$ & 8 & $1175-1200$ \\
\hline$(10,1)$ & 15 & $1350-1375$ \\
\hline$(11,1)$ & 19 & $1450-1475$ \\
\hline$(12,1)$ & * & * \\
\hline$(13,1)$ & 2 & $1025-1050$ \\
\hline$(14,1)$ & 5 & $1100-1125$ \\
\hline$(15,1)$ & 11 & $1250-1275$ \\
\hline$(16,1)$ & 1 & $1000-1025$ \\
\hline $\begin{array}{l}\text { Frequency } \\
\text { Span } \\
\end{array}$ & & 525 \\
\hline $\begin{array}{l}\text { Total } \\
\text { Number Machines/ } \\
\text { Frequencies Required } \\
\text { (Active/Idle) }\end{array}$ & $21+$ & 21 \\
\hline $\begin{array}{l}\text { \# Active } \\
\text { Machines/ } \\
\text { Frequencies } \\
\end{array}$ & 12 & 12 \\
\hline
\end{tabular}


. 
Publlc reporting burden for this collection of information is estimated to average i hour per response, including the time for reviewing instructions, searching existing cata sources, gathering and maintalning the data needed, and completing and reviewing the collection of information. Send comments regarding this burden estimate or any other aspect of this collection of information, including suggestions for reducing this burden, to Washington Headquarters Services, Directorate for information Operations and Reports, 1215 Jefferson Davis Highway, Suite 1204, Arlington, VA 22202-4302, and to the Office of Management and Budget, Paperwork Peduction Project (0704-0188), Washington, DC 20503.
1. AGENCY USE ONLY (Leave blank)
2. REPORT DATE
1992
3. REPOAT TYPE AND DATES COVERED
Technical Memorandum

4. TITLE AND SUBTITLE

5. FUNDING NUMBERS

Achieving Spectrum Conservation for the Minimum-Span and Minimum-

Order Frequency Assignment Problems

6. AUTHOR(S)

WU-643-10-01

Ann O. Heyward

7. PERFoRming ORganization NAME(S) AND ADDRESS(ES)

8. PERFORMING ORGANIZATION REPORT NUMBER

National Aeronautics and Space Administration

Lewis Research Center

Cleveland, Ohio 44135-3191

$E-7010$

9. SPONSORING/MONITORING AGENCY NAMES(S) AND ADDRESS(ES)

10. SPONSORING/MONITORING AGENCY REPORT NUMBER

National Aeronautics and Space Administration

Washington, D.C. 20546-0001

NASA TM -105649

AIAA-92-1960

\section{SUPPLEMENTARY NOTES}

Prepared for the 14th International Communications Satellite Systems Conference sponsored by the American Institute of Aeronautics and Astronautics, Washington, DC, March 22-26, 1992. Responsible person, Ann O. Heyward, (216) 433-3484.

12a. DISTRIBUTION/AVAILABILITY STATEMENT

12b. DISTRIBUTION CODE

Unclassified - Unlimited

Subject Categories 17 and 66

\section{ABSTRACT (Maximum 200 words)}

Effective and efficient solution of frequency assignment problems assumes increasing importance as the radiofrequency spectrum experiences ever-increasing utilization by diverse communications services, requiring that the most efficient use of this resource be achieved. The research presented explores a general approach to the frequency assignment problem, in which such problems are categorized by the appropriate spectrum-conserving objective function, and are each treated as an $\mathrm{N}$-job, $\mathrm{M}$-machine scheduling problem appropriate for the objective. Results obtained and presented illustrate that such an approach presents an effective means of achieving spectrumconserving frequency assignments for communications systems in a variety of environments.

\begin{tabular}{|c|c|c|}
\hline \multicolumn{3}{|c|}{$\begin{array}{l}\text { 14. SUBJECT TERMS } \\
\text { Spectrum; Frequency; Conservation; Scheduling; Minimum-span; Minimum-order }\end{array}$} \\
\hline $\begin{array}{l}\text { 17. SECURTYY CLASSIFICATION } \\
\text { OF REPORT } \\
\text { Unclassified }\end{array}$ & $\begin{array}{l}\text { 18. SECURITY CLASSIFICATION } \\
\text { OF THIS PAGE } \\
\text { Unclassified }\end{array}$ & $\begin{array}{l}\text { 19. SECURTY CLASSIFICATION } \\
\text { OF ABSTRACT } \\
\text { Unclassified }\end{array}$ \\
\hline
\end{tabular}

